\title{
Design of multiligand inhibitors for the swine flu HINI neuraminidase binding site
}

\author{
Manoj M Narayanan ${ }^{1,2}$ \\ Chandrasekhar B Nair ${ }^{2}$ \\ Shilpa K Sanjeeva ${ }^{2}$ \\ PV Subba Rao² \\ Phani K Pullela ${ }^{1,2}$ \\ Colin J Barrow' \\ 'Centre for Chemistry and \\ Biotechnology, Deakin University, \\ Geelong, VIC, Australia; ${ }^{2}$ Bigtec Pvt \\ Ltd, Rajajinagar, Bangalore, India
}

Correspondence: Colin J Barrow Centre for Chemistry and Biotechnology, Deakin University, Locked Bag 20000, Geelong, VIC 3220, Australia

Tel +6I 35227 I3 I8

Fax +6I 352271040

Email cbarrow@deakin.edu.au
This article was published in the following Dove Press journal:

Advances and Applications in Bioinformatics and Chemistry

17 August 2013

Number of times this article has been viewed

\begin{abstract}
Viral neuraminidase inhibitors such as oseltamivir and zanamivir prevent early virus multiplication by blocking sialic acid cleavage on host cells. These drugs are effective for the treatment of a variety of influenza subtypes, including swine flu (H1N1). The binding site for these drugs is well established and they were designed based on computational docking studies. We show here that some common natural products have moderate inhibitory activity for H1N1 neuraminidase under docking studies. Significantly, docking studies using AutoDock for biligand and triligand forms of these compounds (camphor, menthol, and methyl salicylate linked via methylene bridges) indicate that they may bind in combination with high affinity to the H1N1 neuraminidase active site. These results also indicate that chemically linked biligands and triligands of these natural products could provide a new class of drug leads for the prevention and treatment of influenza. This study also highlights the need for a multiligand docking algorithm to understand better the mode of action of natural products, wherein multiple active ingredients are present.
\end{abstract}

Keywords: neuraminidase, influenza, H1N1, multiligand, binding energy, molecular docking, virus

\section{Introduction}

Hemagglutinin and neuraminidase are the two key glycoproteins responsible for viral influenza infection. ${ }^{1}$ Hemagglutinin is present on the surface of the virion and is needed for infection, while neuraminidase is responsible for cleavage of sialic acid (neuraminic acid) from glycans of the infected cell., ${ }^{2,3}$ These two proteins are drug targets for viral infections, and the neuraminidase inhibitors, oseltamivir (Tamiflu ${ }^{\circledR}$, Roche, Basel, Switzerland) and zanamivir (Relenza ${ }^{\circledR}$, Philadelphia, PA, USA), are broad spectrum antiviral drugs, useful for the treatment of a variety of forms of influenza., ${ }^{4,5}$ The World Health Organization recommends the use of oseltamivir or zanamivir for the treatment of H1N1 virus, and patient recovery with these drugs has been impressive. Oseltamivir and zanamivir are reversible competitive inhibitors of neuraminidase, thereby preventing virion release from infected cells. ${ }^{6}$ The binding site interaction of these drugs is well established, and they are amongst only a few drugs with binding sites based on computational docking and quantitative structure-activity relationships that are well understood.

The success of neuraminidase inhibitors can be attributed to the sequence and structure conservation of neuraminidase in all subtypes of influenza. Reports of mutations in neuraminidase are relatively sparse compared with other viral protein domains. ${ }^{1}$ There are significant changes in both hemagglutinin and neuraminidase protein of the 
2012 H1N1 virus, ie, $27.2 \%$ and $18.2 \%$ of the amino acid sequence, respectively, from $2008 \mathrm{H} 1 \mathrm{~N} 1$ isolates. ${ }^{1}$ Though these changes are a complication with respect to the use of existing influenza vaccines, the positive response of this virus to current neuraminidase inhibitors indicates that this change does not significantly alter the protein-ligand interactions.

Hence, neuraminidase is an excellent general target for the control of viral influenza, including H1N1. Although neuraminidase inhibitors such as oseltamivir and zanamivir are approved for both treatment and prevention of influenza, more effective preventive medications would be useful in slowing the spread of H1N1 influenza. ${ }^{4,7}$ Therefore, there is a need to develop improved drugs which can prevent the spread of the virus.

There are several common cold medications which have been available for generations to provide relief from symptoms, including sneezing, blocked nose, sore throat, and nasal congestion. They include over-the-counter products (such as inhalers and vaporizers) and homemade decoctions of spices (eg, ginger, pepper, basil). Most of these have a pungent aromatic odor and the exact mechanism of action of their active constituents is unknown. The effectiveness of these compounds in relieving symptoms caused by influenza warrants further investigation into whether any of this efficacy comes from specific binding mechanisms. If these compounds selectively bind to specific targets then they could potentially be used more broadly in H1N1 influenza prevention or treatment.

\section{Materials and methods}

The Protein Data Bank crystal structure (2hu0) of the neuraminidase in $\mathrm{H} 5 \mathrm{~N} 1$ bound to oseltamivir was used as a reference ${ }^{8}$ and a model built for H1N1 neuraminidase was used for docking studies. ${ }^{9}$ AutoDock ${ }^{10}$ (The Scripps Research Institute, La Jolla, CA, USA) was used for protein-ligand docking studies. Chemical structures were downloaded from Pubchem and similar databases and verified or drawn using ACD Chemsketch (Advanced Chemistry Development, Inc., Toronto, ON, Canada) Protein electrostatic potential was calculated using the Adaptive Poisson Boltzmann Solver (ABPS) with AutoDockTools release 1.5.4. ${ }^{10}$ Molecular graphics images were produced using the UCSF Chimera package from the Computer Graphics Laboratory, University of California, San Francisco, CA, USA. ${ }^{11}$

\section{Results and discussion \\ Neuraminidase structure selection and protein model}

Maurer-Stroh et al have demonstrated that the neuraminidase present in current variants of $\mathrm{H} 1 \mathrm{~N} 1$ is similar to that present in $\mathrm{H} 5 \mathrm{~N} 1$ avian influenza. ${ }^{9}$ The crystal structure of neuraminidase in $\mathrm{H} 5 \mathrm{~N} 1$ bound to oseltamivir is known and we used this as a reference structure for our study. MaurerStroh et al built a model for H1N1 based on the previously reported information for $\mathrm{H} 5 \mathrm{~N} 1$, and we used the same model after ensuring that the crystal structure of neuraminidase in $\mathrm{H} 5 \mathrm{~N} 1$ and $\mathrm{H} 1 \mathrm{~N} 1$ is similar (root-mean-square deviation $0.81 \AA$ ). Figure 1 shows the overlay of H5N1 and H1N1 neuraminidase structures. Recent crystal structures of some strains of H1N1 neuraminidase, such as the 1918 influenza viral strain, indicate that there is a high degree of structural similarity to the H5N1 strain. Both are group 1 subtypes characterized by a similar conformation for the 150 loop or 150 cavity which, on binding to zanamivir, undergoes conformational change that is similar for both $\mathrm{H} 1 \mathrm{~N} 1$ and H5N1 subtypes. ${ }^{12}$

\section{Binding site of HINI neuraminidase}

The binding site of neuraminidase is a conserved region in different variants of influenza A and B. The key step of viral attack and multiplication in the host cell is driven by binding of viral neuraminidase to sialic acid on the human cell surface. ${ }^{3}$ Hence, the inhibitors of neuraminidase (oseltamivir and zanamivir) are designed in such a way that they are chemically as similar as possible to sialic acid, with improved enzyme affinity. Figure 2 shows the binding site of H1N1 neuraminidase, depicting the two different electrostatic grooves as A and B, representing two complementary regions, a highly negatively charged region (depicted by red color), and a positively charged stretch. Under docking condi-

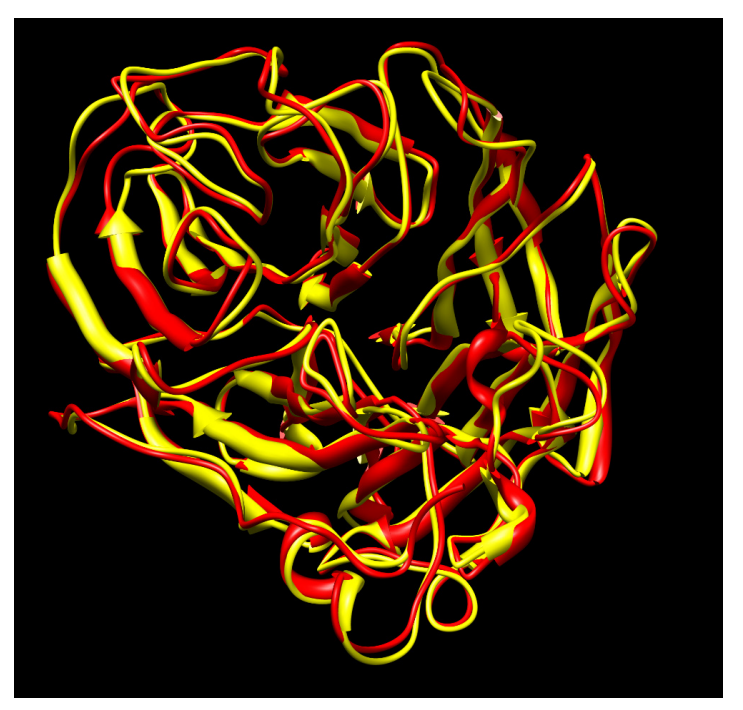

Figure I Overlay of $\mathrm{H} 5 \mathrm{NI}$ crystal structure (2hu0, yellow) and the modeled structure of $\mathrm{HINI}$ (red). The root-mean-square deviation of the two structures is $0.8 \mathrm{I} \AA$. 


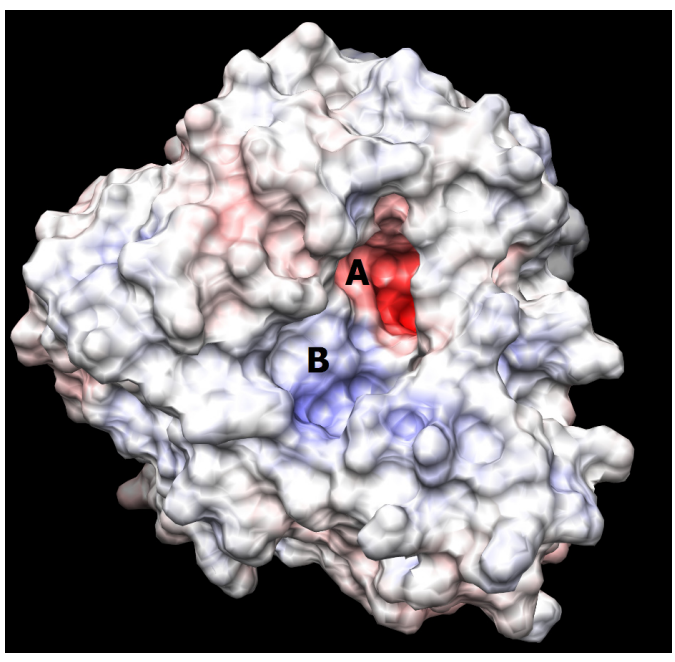

Figure 2 Picture depicting the two regions of the HINI neuraminidase binding site. The red region $(\mathbf{A})$ is the electronegative region, where the amine-rich functional groups of ligands bind, and the large blue region (B) a large electropositive zone. The deep binding pocket (A) could host many kinds of ligands, but high affinity can be obtained only when there is a well defined functional group interaction (electrostatic and/or structural).

tions, sialic acid has the possibility of nine hydrogen bonds at the A binding site and synthetic inhibitors like oseltamivir and zanamivir have about seven. Important aspects for the H1N1 neuraminidase quantitative structure-activity relationship are the electrostatic interaction between ligand and protein, and the possible formation of salt bridges between the amine-rich terminus of the ligand and glutamic acid in the binding site. This kind of ligand design is dependent on interaction between the binding site amino acid residues and the ligand, and is probably a reason why oseltamivir-resistant strains have started to evolve. ${ }^{13-15}$

\section{Binding of oseltamivir and sialic acid to HINI neuraminidase}

Oseltamivir is one of the few inhibitors of neuraminidase with subnanomolar affinity and currently in clinical use for the treatment of influenza. ${ }^{16}$ In our docking mode, oseltamivir is the strongest ligand of those studied (Table 1), with the lowest docking energy of $-13.94 \mathrm{kcal} / \mathrm{mol}$. The predicted binding constant for oseltamivir is at least 10 times higher than that for sialic acid (Figure 3). The hydrogen bond-rich interaction of sialic acid has not resulted in higher binding affinity. The higher affinity of oseltamivir is probably related to salt bridge formation (Figure 4). In our analysis, zanamivir had a binding affinity similar to that of sialic acid, with docking energy of $-12.84 \mathrm{kcal} / \mathrm{mol}$ (Table 1 ).

Zanamivir also forms a binding site salt bridge and has seven hydrogen bonds possible at the binding site. The significantly higher binding affinity of oseltamivir as compared
Table I Docking energies (affinity) for different chemicals with HINI neuraminidase

\begin{tabular}{|c|c|c|}
\hline & Compound name & $\begin{array}{l}\text { Docking energy } \\
\text { (kcal/mol) }\end{array}$ \\
\hline I & Oseltamivir & -13.94 \\
\hline 2 & Zanamivir & -12.84 \\
\hline 3 & Methyl salicylate-menthol-camphor & -12.68 \\
\hline 4 & Sialic acid & -12.47 \\
\hline 5 & Camphor-methyl salicylate-menthol & -12.02 \\
\hline 6 & Oleanolic acid & -11.74 \\
\hline 7 & Rosmarinic acid & $-|1.7|$ \\
\hline 8 & Methyl salicylate-camphor-menthol & -11.18 \\
\hline 9 & Carnosic acid & -10.49 \\
\hline 10 & Capsaicin & -10.3 \\
\hline 11 & Gingerol & -9.97 \\
\hline 12 & Rosmanol & -9.67 \\
\hline 13 & Camphor-menthol & -9.31 \\
\hline 14 & Chlorogenic acid & -9.09 \\
\hline 15 & Caryophyllene & -8.51 \\
\hline 16 & Piperine & -8.43 \\
\hline 17 & Zingiberene & -7.81 \\
\hline 18 & Geraniol & -7.06 \\
\hline 19 & Hydroxycitric acid & -6.98 \\
\hline 20 & Bisabolol & -6.64 \\
\hline 21 & $\beta$-Terpineol & -6.62 \\
\hline 22 & Borneol & -6.61 \\
\hline 23 & Eugenol & -6.45 \\
\hline 24 & Linalool & -6.27 \\
\hline 25 & Ascorbic acid & -6.23 \\
\hline 26 & Camphor & -6.15 \\
\hline 27 & Thymol & -6.14 \\
\hline 28 & Cineole & -6.12 \\
\hline 29 & Methyl salicylate & -5.8 \\
\hline 30 & $\gamma$-Terpinene & -5.74 \\
\hline 31 & Menthol & -5.73 \\
\hline 32 & Thymoquinone & -5.39 \\
\hline 33 & Cinnamaldehyde & -5.32 \\
\hline
\end{tabular}

with zanamivir, even though both have a similar number of hydrogen bonds and form salt bridges, indicates that there may be a structural parameter that impacts binding that was not determined in previous studies. ${ }^{14,15,17}$

\section{Docking of natural product chemical constituents}

We docked the three major chemical constituents of common inhalers, namely menthol, camphor, and methyl salicylate. These components as well as antioxidants (rosemary oil components), traditional cold medicines (ginger, pepper) and common natural essential oil products have shown significant affinity for H1N1 neuraminidase (Table 1). Surprisingly, we observed that a steroid molecule (oleanolic acid) present in rosemary extract has high affinity for H1N1 neuraminidase 


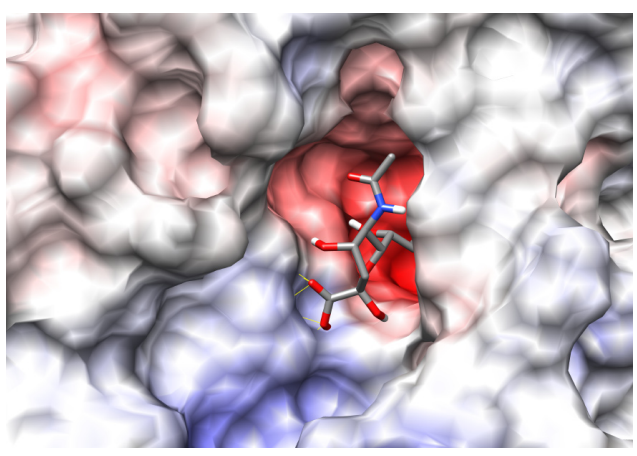

Figure 3 Model showing docked configuration of sialic acid with HINI neuraminidase.

$(-11.74 \mathrm{kcal} / \mathrm{mol})$. Oleanolic acid does not bind with as many hydrogen bonds as sialic acid and oseltamivir, indicating that it has a structural specificity for the neuraminidase binding site. Cold and influenza relief recipes used in traditional Indian medicine are general decoctions of herbs and often use cocktails containing multiple ingredients. ${ }^{18,19}$ These would have multiple active components, which may have cumulative or synergistic actions. Based on this, we evaluated the binding of some of these compounds in combination. Since the docking software accepts only one molecule at a time, we evaluated the combination effect using a "linkedstructure" approach. As seen in Table 1, these combination linked structures had significantly higher binding affinities than the individual molecules.

\section{Multiligand binding: linked structure approach}

Of the natural products evaluated, the most potent H1N1 neuraminidase binders in our model were oleanolic acid and rosmarinic acid. It is possible that multiple components could simultaneously bind to the of H1N1 neuraminidase. AutoDock does not allow multiple ligands to be docked with the same protein at the same time. To the best of our knowl-

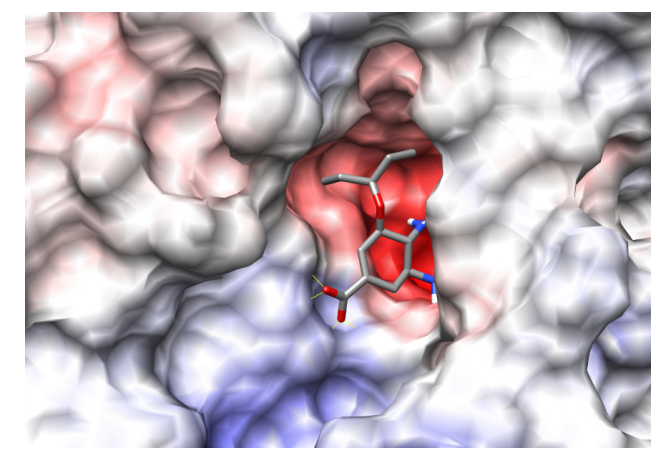

Figure 4 Model showing docked configuration of oseltamivir with HINI neuraminidase. edge, there is no docking program which allows multiple ligand docking. Simultaneous binding of ligands to the same target is an unusual concept, but it has been used in biligand design for some protein targets, such as oxidoreductases. ${ }^{20}$ This approach uses docking and nuclear magnetic resonance studies for two compounds binding next to each other, using NOE determined distances to show the spatial relationship between each compound. A new compound containing both molecular structures can then be synthesized and tested for binding affinity. This biligand approach has resulted in some clinical leads and the linking of two active compounds through methylene groups has shown some success. In the case of H1N1 neuraminidase, the binding site is so large that it can readily accommodate more than one ligand. Neuraminidase is similar to oxidoreductase in that both have two binding sites A and B adjacent to each other. ${ }^{21}$

We hypothesized that if a ligand was structurally connected through a methylene group at the methyl terminus of a molecule it should provide a dockable biligand with sufficient flexibility due to two additional single bonds, which could mimic the simultaneous binding of two ligands. Among the functional groups commonly present in both natural and synthetic inhibitors, methyl groups are physiologically the least relevant due to their small size and lack of chemical functionality. Methylene linking has been successfully used in biligand development for oxidoreductases and binding shown to occur using nuclear magnetic resonance. The methylene group itself has minimal impact on binding and the methylenes primarily enable flexibility in orientation of the biligands. Hence, conjugation to a terminal methyl group may not significantly change the affinity of the original ligand. Secondly, when two molecules are chemically linked, the degrees of freedom for rotation decreases, which could affect the docking results, in that docking software may not be able to test all possible orientations. By bridging methyl groups of ligands with a methylene group, we created two extra rotatable bonds, which compensates for the loss of degrees of freedom. Finally, introduction of an extra methylene functional group creates an average $3 \AA$ distance between molecules, which is the minimum distance two molecules are expected to maintain when they are bound together in solid state to a target.

The first combination ligand we designed was camphormenthol. This molecule is predicted to bind with a docking energy of $-9.31 \mathrm{kcal} / \mathrm{mol}$, which is about 1000 -fold higher than the affinity of each individual chemical component. This combination molecule formed only one hydrogen bond, indicating that structural affinity rather than electrostatic interaction is 


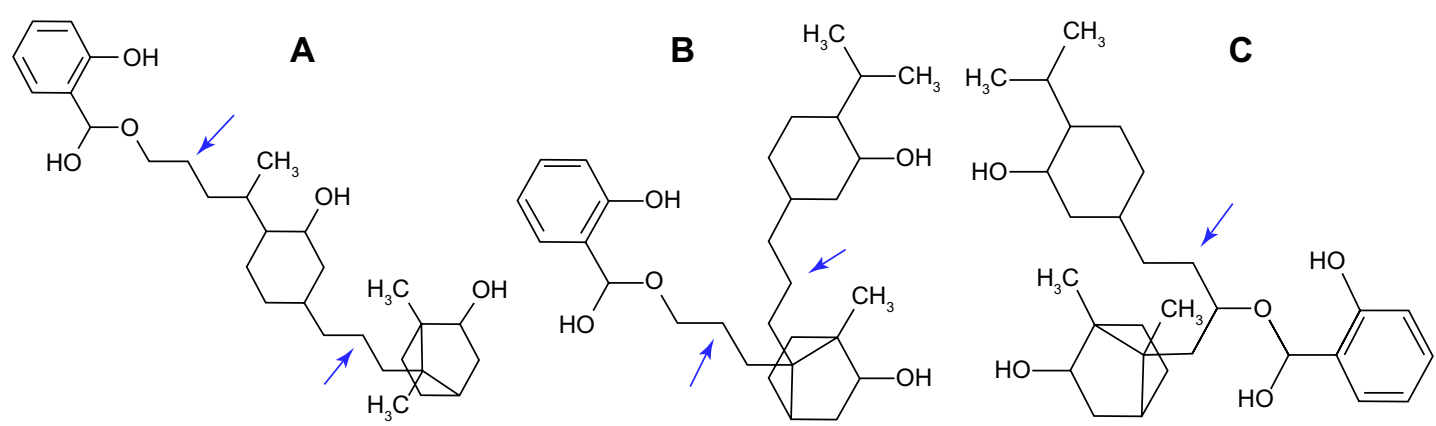

Figure 5 Chemical structure of combination ligands. Methyl salicylate-menthol-camphor (A), methyl salicylate-camphor-menthol (B), and camphor-methyl salicylate-menthol $(\mathbf{C})$. The arrows show the methylene bridges artificially created to enable the possibility of docking the combination ligand with the enzyme. Note the drop in docking energy of $\mathbf{B}(-11.18 \mathrm{kcal} / \mathrm{mol})$ when menthol and camphor position is interchanged from $\mathbf{A}(-12.68)$.

primarily responsible for the increased binding affinity. We then designed a triligand comprising menthol, camphor, and methyl salicylate, with three possible structural forms. We followed the methylene linking strategy in designing these molecules, and they were methyl salicylate-menthol-camphor, methyl salicylate-camphor-menthol, and camphor-methyl salicylatementhol (Figure 5). We observed that all three combination ligands had good affinity for H1N1 neuraminidase, but the mode and orientation of binding was different. Among these, methyl salicylate-menthol-camphor had the highest affinity, which was almost the same as sialic acid and zanamivir (Table 1). The binding of methyl salicylate-menthol-camphor resembles a triangular structure covering the electronegative pocket in the binding region (Figure 6). Importantly, the methyl salicylate-menthol-camphor is bound completely at the A site and no part of the molecule was interacting with the B site. This indicates that the interaction of this ligand with H1N1 neuraminidase has a strong structural basis.

In the case of methyl salicylate-camphor-menthol (Figure 7), the menthol group occupied the B site. This is in contrast with methyl salicylate-menthol-camphor, where there was a specific stabilizing interaction with the A binding site (Figure 6). This specific interaction was absent with

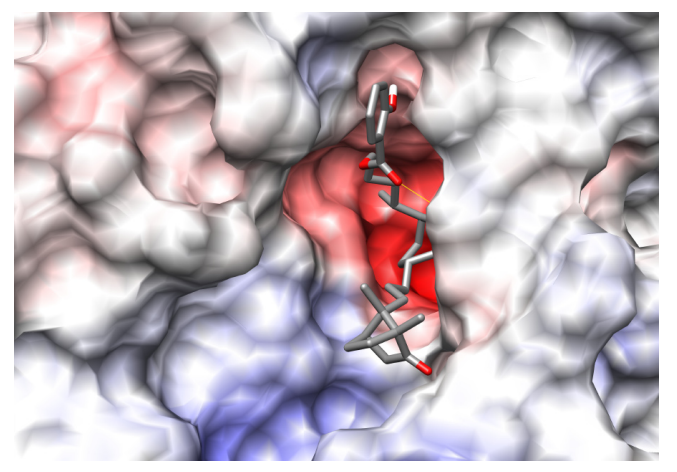

Figure 6 Docking of methyl salicylate-menthol-camphor to neuraminidase enzyme. methyl salicylate-camphor-menthol and may be responsible for the lower docking energy of this triligand ( -11.18 versus $-12.68 \mathrm{kcal} / \mathrm{mol}$, refer to Table 1$)$.

The difference in affinity of other combination ligands with respect to methyl salicylate-menthol-camphor suggests that there could be specific structural sites for the three ligands, and interchanging their position in the binding site alters the overall binding affinity.

\section{Simultaneous reversible binding of multiple ligands}

Docking studies are a good initial method of analysis that can provide binding information for these complex natural products. These ligands can bind reversibly to H1N1 neuraminidase, with affinity similar to sialic acid, indicating that H1N1 neuraminidase attack on host cells is likely to be inhibited. Current docking algorithms do not allow multiple-ligand simultaneous docking to target protein. Most natural products are known to have multiple active components, providing cumulative or synergistic effects, also overcoming buildup of resistance. It is highly desirable to have new molecular docking software capable of multiligand docking, for analyzing and understanding the mode of action of natural products.

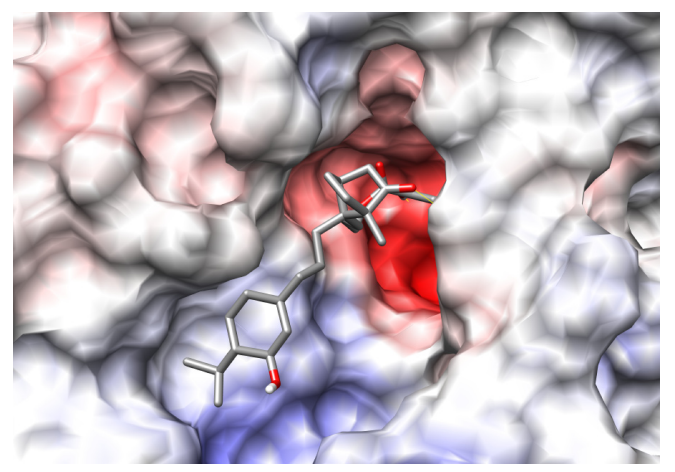

Figure 7 Docking of methyl salicylate-camphor-menthol with neuraminidase. 


\section{Possible experimental verification of the combination ligand hypothesis}

The neuraminidase enzyme is a well studied system, and protocols for its expression, purification, and crystallization have been reported in the literature. ${ }^{22}$ The binding of multiple ligands could be verified by crystallizing neuraminidase in the presence of varying ratios of these constituents, where difficulties in crystallization due to high volatility would need to be overcome. Synthesis of the larger and probably less volatile biligands and triligands and their cocrystallization with H1N1 neuraminidase could also be tested experimentally. It is likely that some of the increased binding energy observed for biligands and triligands is due to increased ligand size, resulting in an increased hydrophobic contact surface area, so synthesis and experimental verification of H1N1 neuraminidase binding is particularly important to validate the potential of these ligands as drug leads. Alternatively, clinical evaluation would give insight into the benefits of these types of potential medications. Given that compounds like oleanolic acid, rosmarinic acid, methyl salicylate, menthol, and camphor are commonly used ingredients with low toxicity, there would be minimal concern regarding safety and adverse effects during a clinical study. It is possible that observation and population studies would provide useful initial clinical information on any protective effect of these compounds.

\section{Conclusion}

Modeling studies of biligands and triligands, created by linking methyl salicylate, menthol, and camphor via methylene bridges, indicate that in combination these compounds may be potential inhibitors of H1N1 neuraminidase. It is important to note, however, that high docking energies are indicative but not necessarily predictive of binding affinity. Therefore, these compounds need to be synthesized and tested experimentally to determine if they in fact have high binding affinity for H1N1 neuraminidase.

We have shown that the strong hydrogen bond-driven affinity of zanamivir, sialic acid, and oseltamivir is not the mechanism for methyl salicylate-menthol-camphor binding. The hydrophobic and structural interaction of these natural products indicates sequential and reversible binding to H1N1 neuraminidase. The structural and hydrophobic interactions of these chemical constituents offer the possibility of activity against H1N1 neuraminidase. By designing biligands and triligands, we were able to use AutoDock to study multiligand binding. A combination of such small molecules or these biligands and triligands may offer a new class of lead drug candidates for the prevention or treatment of influenza.

\section{Acknowledgment}

This research was supported by a grant from the Indian Council of Medical Research.

\section{Disclosure}

The authors report no conflicts of interest in this work.

\section{References}

1. Gallaher WR. Towards a sane and rational approach to management of influenza H1N1. Virol J. 2009;6:51-57.

2. Harrison SC. Viral membrane fusion. Nat Struct Mol Biol. 2008;15: 690-698.

3. Burmeister WP, Ruigrok RWH, Cusack S. The 2.2 A resolution crystal structure of influenza $B$ neuraminidase and its complex with sialic acid. EMBO J. 1992;11:49-56.

4. Heinonen S, Silvennoinen H, Lehtinen P, et al. Early oseltamivir treatment of influenza in children 1-3 years of age: a randomized controlled trial. Clin Infect Dis. 2010;51:887-894.

5. Crusat M, de Jong MD. Neuraminidase inhibitors and their role in avian and pandemic influenza. Antivir Ther. 2007;12:593-602.

6. De Clercq E. Antiviral agents active against influenza A viruses. Nat Rev Drug Discov. 2006;5:1015-1023.

7. Eiland LS, Eliand EH. Zanamivir for the prevention of influenza in adults and children age 5 years and older. Ther Clin Risk Manag. 2007;3:461-465.

8. Russell RJ, Haire LF, Stevens DJ, et al. The structure of H5N1 avian influenza neuraminidase suggests new opportunities for drug design. Nature. 2006;443:45-49.

9. Maurer-Stroh S, Ma J, Lee RT, Sirota FL, Eisenhaber F. Mapping the sequence mutations of the $2009 \mathrm{H} 1 \mathrm{~N} 1$ influenza A virus neuraminidase relative to drug and antibody binding sites. Biol Direct. 2009;4:1-9.

10. Morris GM, Huey R, Lindstrom W, et al. Autodock4 and AutoDockTools4: automated docking with selective receptor flexibility. J Comput Chem. 2009;30:2785-2791.

11. Pettersen EF, Goddard TD, Huang CC, et al. UCSF chimera - a visualization system for exploratory research and analysis. J Comput Chem. 2004;25:1605-1612.

12. Xu X, Zhu X, Dwek RA, Stevens J, Wilson IA. Structural characterization of the 1918 Influenza Virus H1N1 neuraminidase. JVirol. 2008;82: 10493-10501

13. Wang NX, Zheng JJ. Computational studies of H5N1 influenza virus resistance to oseltamivir. Protein Sci. 2009;18:707-715.

14. Collins PJ, Haire LF, Lin YP, et al. Crystal structures of oseltamivirresistant influenza virus neuraminidase mutants. Nature. 2008;453: $1258-1261$.

15. Lin L, LiY, Zhang L, Hou T. Theoretical studies on the susceptibility of oseltamivir against variants of $2009 \mathrm{~A} / \mathrm{H} 1 \mathrm{~N} 1$ influenza neuraminidase. J Chem Inf Model. 2012;52:2715-2729.

16. Schirmer P, Holodniy M. Oseltamivir for treatment and prophylaxis of influenza infection. Expert Opin Drug Saf. 2009;8:357-371.

17. Han N, Liu X, Mu Y. Exploring the mechanism of zanamivir resistance in a neuraminidase mutant: a molecular dynamics study. PLoS One. 2012;7:e44057.

18. Lee SJ, Umano K, Shibamoto T, Lee KG. Identification of volatile components in basil (Ocimum basilicum L.) and their antioxidant properties. Food Chem. 2005;91:131-137.

19. Ballabh B, Chaurasia OP. Traditional medicinal plants of cold desert Ladakh: used in treatment of cold, cough and fever. J Ethnopharmacol. 2007;112:341-349. 
20. Sem DS, Bertolaet B, Baker B, et al. Systems-based design of bi-ligand inhibitors of oxidoreductases: filling the chemical proteomic toolbox. Chem Biol. 2004;11:185-194.

21. Colman PM. Influenza virus neuraminidase: structure, antibodies, and inhibitors. Protein Sci. 1994;3:1687-1696.
22. Schmidt PM, Attwood RM, Mohr PG, Barrett SA, McKimmBreschkin JL. A generic system for the expression and purification of soluble and stable influenza neuraminidase. PLoS One. 2011;6:1-13.

\section{Publish your work in this journal}

Advances and Applications in Bioinformatics and Chemistry is an international, peer-reviewed open-access journal that publishes articles in the following fields: Computational biomodeling; Bioinformatics; Computational genomics; Molecular modeling; Protein structure modeling and structural genomics; Systems Biology; Computational Biochemistry;
Computational Biophysics; Chemoinformatics and Drug Design; In silico $\mathrm{ADME} /$ Tox prediction. The manuscript management system is completely online and includes a very quick and fair peer-review system, which is all easy to use. Visit http://www.dovepress.com/testimonials.php to read real quotes from published authors.

Submit your manuscript here: http://www.dovepress.com/advances-and-applications-in-bioinformatics-and-chemistry-journal 